\title{
CHEMICAL COMPOSITION OF NOVA CYGNI 1975
}

A. A. Boyarchuk

Crimean Astrophysical observatory, USSR

Abstract: The chemical composition of Nova Cygni 1975 was determined by a curve-of-growth analysis. A strong excess of hellum and the CNO -group abundances and a normal $\mathrm{Si}$ abundance was found.

Spectral observations of N Cyg 1975 were begun at the Crimean Astrophysical observatory on 29 August 1975 , at $18^{\mathrm{h}} 50^{\mathrm{m}}$ UT, one day before light maximum (Boyarchuk et al. 1977). Twelve spectrograms with a dispersion of 4,6 and $12 \AA / \mathrm{mm}$ were taken during this night. The spectral type of the Nova was $\mathrm{B} 2 \mathrm{Ia}^{+}$and no emissions appeared in the spectrum. During the following night, 10 spectrograms were obtained. The spectral type of the Nova was $\mathrm{A}_{2} \mathrm{Ia}^{+}$qe, and strong emission lines with P Cygni-type profiles could be detected. Since absorption lines were not observed on 30 August, only the spectrograms taken on 29 August can be used for a curve-of-growth analysis.

Fig. 1 shows the tracing of a $4 \AA / \mathrm{mm}$ spectrogram. All absorption lines are very broad, with total width of $920 \mathrm{~km} / \mathrm{sec}$. Many lines are therefore blended and make the analysis difficult. But since the line-blending is mainly caused by macromotion, the equivalent width of the blends is equal to the sum of the equivalent widths of the components and the blends can be separated. These equivalent widths were then used for a determination of the atmospheric parameters and the chemical composition. The gf-values were taken from wiese et al. (1966) and wiese et al. (1969). The theoretical curveof-growth was used for $B_{1} / B_{0}=4 / 3$ (Wrubel 1949). The observed curve-of-growth is shown in Fig. 2. For the determination of the excitation temperature OII-lines were used. We assume that $\theta_{i o n}$ $=0.92 \theta_{\text {ex }}$.

The results are given in Table 1 . The nova envelope has a high turbulent velocity of $60 \mathrm{~km} / \mathrm{sec}$, which is not surprising since we have to deal with an explosion.

It should be noted that we have the same situation for all elements: most of the atoms are in an ionization stage that is one stage higher than the observed one. An error in the electron pressure has therefore no effect on the determination of the chemical composition. 


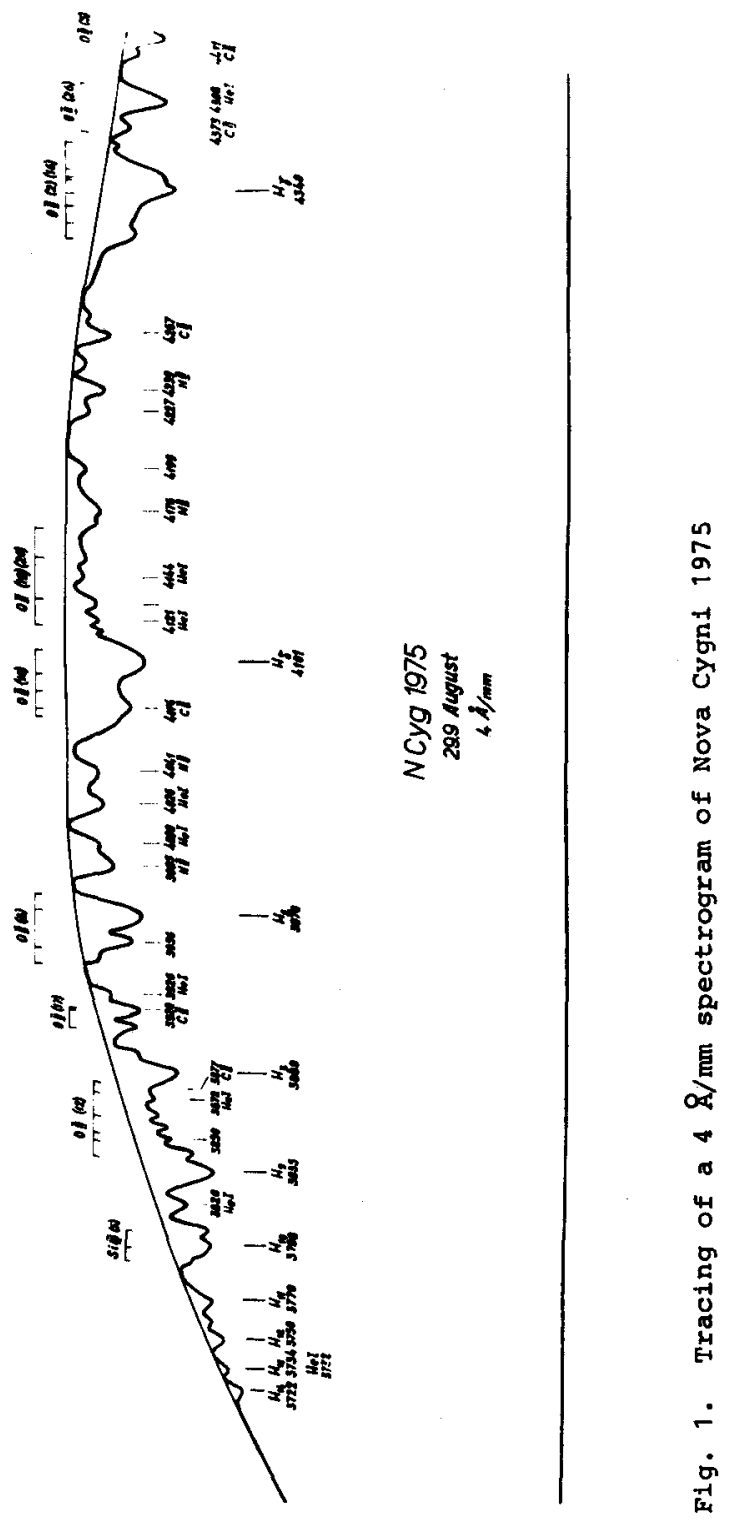




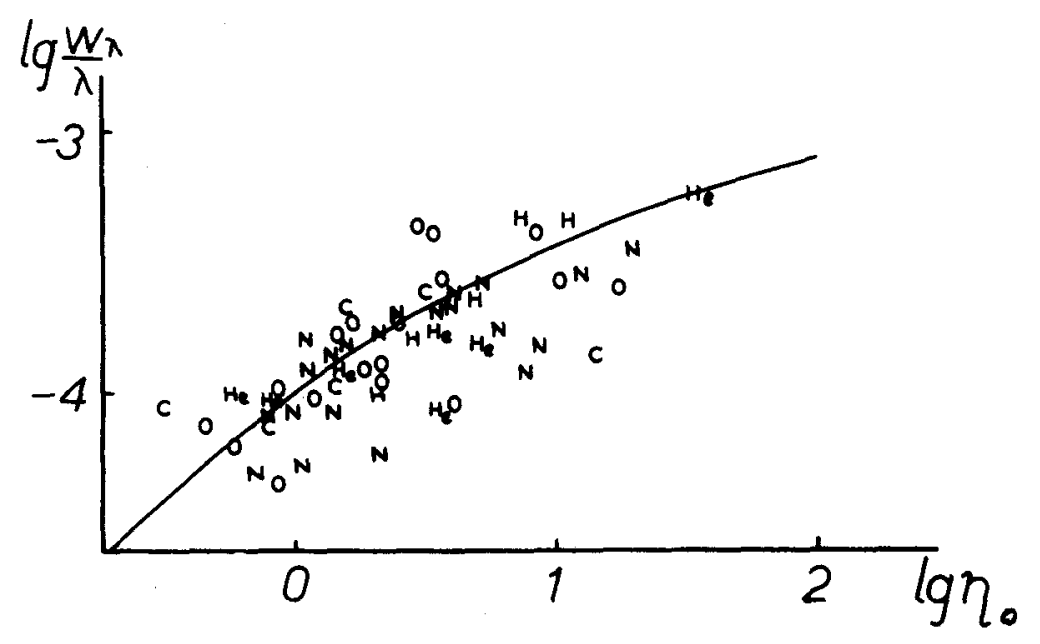

Fig. 2. Observed curve-of-growth.

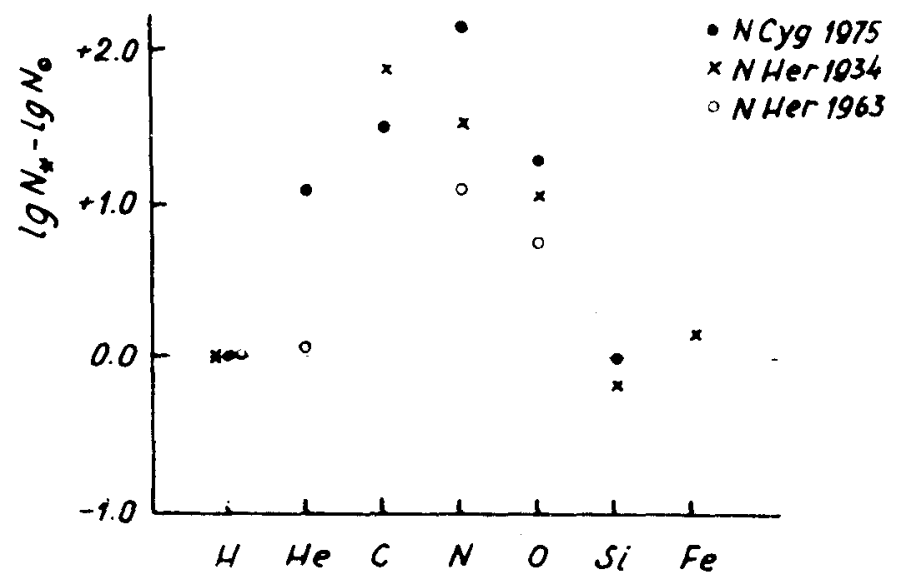

Fig. 3. The chemical composition of N Cyg 1975, N Her 1934, and $\mathbf{N}$ Her 1963 relative to that of the sun. 
Table 1.

Physical Properties of Nova Cygni 1975

\begin{tabular}{lc}
\hline & N Cyg 1975 \\
\hline$\theta_{\text {ex }}$ & 0.25 \\
$\theta_{\text {ion }}$ & $0.92 \theta_{\text {ex }}=0.23$ \\
$\lg \mathrm{p}_{\mathrm{e}}$ & +0.85 \\
$\operatorname{lg~k}$ & -24.0 \\
$\operatorname{lg~} \mathrm{v}_{t}$ & 1.78 \\
\hline
\end{tabular}

Table 2.

Chemical Composition of Nova Cygni 1975

\begin{tabular}{lrr}
\hline & N CYg 1975 & Sun \\
\hline H & $12.00(4)$ & 12.00 \\
He & $12.10(7)$ & 10.93 \\
C & $10.00(6)$ & 8.52 \\
N & $10.30(20)$ & 7.96 \\
O & $10.10(20)$ & 8.82 \\
Si & $7.50(4)$ & 7.52 \\
\hline
\end{tabular}

The number of lines used in the analysis is given in brackets.

The chemical composition (Table 2) is very peculiar. Helium, carbon, nitrogen and oxygen are overabundant in the envelope of N Cyg 1975 as compared to the sun. Such chemical composition peculiarities are typical for novae. Fig. 3 shows the chemical composition of N Cyg 1975 relative to that of the sun together with the data for N Her 1934 and N Her 1963, which have been obtained earlier in the Crimean Astrophysical Observatory (Bortash and Boyarchuk 1965, Mustel and Boyarchuk 1958).

\section{References:}

Bortash, T.M., Boyarchuk, A.A., 1965, Izv. Crimean Aph. Obs. 33, 173. Boyarchuk, A.A., Galkina, T.S., Gershberg, R.E., Krasnobabtsev, V.I., Rachkovskaya, T.M., Shakhovskaya, N.I., 1977, Astr. Z. 54, 458. 
Mustel, E.R., Boyarchuk, M.E., 1959, Astr. Z, 34, 762.

Wiese, W.L., Smith, M.W., Glennon, B.M., 1966, NSRDS-NBS $\underline{4}$.

Wiese, W.L., Smith, M.W., Miles, B.M., 1969, NSRDS-NBS 22.

Wrubel, M.н., 1949, Ap. J., 7, 109, 66.

D I S CUSSI ON Of paper by BOYARCHUK:

WOLF: The temperature of $20,000 \mathrm{~K}$ seems rather high. From infrared observations of Gallagher and Ney which started very early, a temperature of the expanding photospheric shell of about $10,000 \mathrm{~K}$ is indicated.

BOYARCHUK: The temperature of Nova decreased very rapidly. During our first observations, taken on 29 August at $19 \mathrm{~h}$ UT, the temperature was $18,000-20,000^{\circ} \mathrm{K}$. This value was determined by analyzing the OII-lines and the energy distribution in the spectral region 3300-7500A. The spectrum of Nova showed lines of $\mathrm{H}, \mathrm{HeI}, \mathrm{CII}, \mathrm{OII}, \mathrm{NII}, \mathrm{S}$ IIII and corresponds to B2Ia. The next set of spectrograms was taken on 30th August at $17 \mathrm{~h}$ UT. At that time the Nova had a spectral type of A2Ia, and its temperature was less than $10,000^{\circ} \mathrm{K}$. 